\title{
LOW ENERGY SPUTTERING EXPERIMENTS FOR ION ENGINE LIFETIME ASSESSMENT
}

\author{
Olivier B. DUCHEMIN" and James E. POLK ${ }^{+}$
}

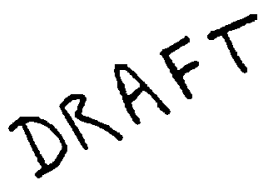

\author{
Jet Propulsion Laboratory \\ California Institute of Technology \\ Pasadena, CA 91109-8099
}

\begin{abstract}
The sputtering yield of molybdenum under xenon ion bombardment was measured using a Quartz Crystal Microbalance. The measurements were made for ion kinetic energies in the range $100-1 \mathrm{keV}$ on molybdenum films deposited by magnetron sputtering in conditions optimized to reproduce or approach bulk-like properties. SEM micrographs for different anode bias voltages during the deposition are compared, and four different methods were implemented to estimate the density of the molybdenum films. A careful discussion of the Quartz Crystal Microbalance is proposed and it is shown that this method can be used to measure mass changes that are distributed unevenly on the crystal electrode surface, if an analytical expression is known for the differential mass-sensitivity of the crystal and the erosion profile. Finally, results are presented that are in good agreement with previously published data, and it is concluded that this method holds the promise of enabling sputtering yield measurements at energies closer to the threshold energy in the very short term.
\end{abstract}

\section{Introduction}

After the generation of flyby missions flown by NASA during the last decades and the first orbiter missions, space exploration has now moved on to newgeneration missions involving orbiters, landers and sample-returns. These missions, are increasingly demanding in terms of propulsion needs, and increasingly long lifetimes are required for electric propulsion devices as they evolve into mature candidates for primary propulsion of interplanetary probes.

As an example, the NASA Solar Electric Propulsion (SEP) Technology Application Readiness (NSTAR) thruster flown on the mission Deep Space 1 has a maximum demonstrated throughput of $83 \mathrm{~kg}$ of xenon propellant, while future missions such as Science and Technology (ST) 4, a comet rendezvous, or negative-C3 launches of interplanetary probes may call for thruster evolutions with a maximum propellant throughput of, respectively, about 120 and $180 \mathrm{~kg}$. In order to contribute to these advances, the NSTAR program has been supporting both testing and analysis activities. The latter require a good understanding of the ion-erosion processes in ion engines and knowledge of the sputtering yield (expected number of atoms removed per incident ion) as a function of ion kinetic energy, for various materials making up the engine components subject to ion bombardment. These parts are, primarily, the screen grid, the starter electrode and cathode components, while the materials of interest include molybdenum, tantalum and tungsten. Further work may be extended to measurements on silicon, silicon nitride and silicon oxide for applications to micropropulsion.

Data for xenon ion energies down to $100 \mathrm{eV}$ have been published by Weijsenfeld, Hoogendoorn and Koedam, ${ }^{1}$ Rosenberg and Wehner, ${ }^{2}$ and Stuart and Wehner. ${ }^{3}$ More recently, Bhattacharjee et al. ${ }^{4}$ published data for $\mathrm{Xe}^{+}$ions on $\mathrm{Mo}$ in the energy range $150-600$ $\mathrm{eV}$. This paper presents experimental results for ion energies ranging from $1 \mathrm{keV}$ down to $100 \mathrm{eV}$ for xenon ions on molybdenum, and describes an ongoing effort to be extended to both lower energies and the other materials of interest in the coming months.

\footnotetext{
- Graduate Student, Aeronautical Engineering AIAA Student Member

'Group Supervisor, Advanced Propulsion Technology Group.
}

Copyright 1999 by the American Institute of Aeronautics and Astronautics. Inc. No copyright is asserted in the United States under Tite 17, U.S. Code. The US. Government has a royalty-free license to exercise all rights under the copyright claimed herin for Governmental Purposes. All other rights are reserved by the copyright owner. 


\section{The Quartz Crystal Microbalance (OCM)}

\section{Overview}

The use of a single crystal quartz electromechanical resonator to measure sputtering yields was described as early as 1961 by McKeown, ${ }^{5}$ and was further demonstrated by Hayward and Wolter ${ }^{6}$ and Andersen and Bay. ${ }^{7}$ The QCM is a device for the measurement of the areal mass density of thin films that presents the advantages of being relatively convenient to use, highly sensitive, direct, and to make possible dynamic, in situ measurements of absolute data. ${ }^{8,9}$ The main difficulty with this method is the requirement that the sample material be deposited as a thin layer a few microns thick on a quartz crystal substrate. It is also commonly believed that correct use of this method mandates a uniform dose of incident ions on the $1.4-\mathrm{cm}$ diameter sample surface, but it will be shown here how additional care can be taken so that not only this limitation does not hold, but in fact a significant advantage can be gained from focusing the beam on a spot a few millimeters in diameter.

In addition, significant and recent technical improvements have made the QCM technique even more attractive, ${ }^{10,11}$ with advances pertaining to the oscillator (shape of the crystal and electrodes, electrode to quartz bonding), the transducer (supporting environment of the quartz), and perhaps even more significantly to the electronics: the use of the microprocessor and modern electronics allow "intelligent" measurement systems to not only determine the resonant frequency but also verifies that the crystal is oscillating in the desired mode, greatly increasing the speed, accuracy and precision (to better than 1 part in $10^{8}$ ) of the instrument. ${ }^{10}$

\section{Principle}

Sauerbrey ${ }^{12}$ first expressed the relationship between the change in the resonant frequency of a coated crystal oscillator $\Delta f$, to the change in areal mass density at its surface $\Delta \sigma$, as

$$
\Delta \sigma / \sigma_{0}=-\Delta f / f_{0}
$$

where $\sigma_{o}$ is the initial areal mass density and $f_{o}$ is the initial resonant frequency of the oscillator. In the case of a planar resonator, as shown in Fig. 1, it can be seen that the positions $:= \pm h$ are antinodes for the first thickness-shear mode of oscillations, so that the thickness $2 h$ of the crystal corresponds to half a wavelength for this mode and we can write

$$
2 h=\frac{C_{A T} / 2}{f_{0}}
$$

where $C_{A T}$ is the wavespeed for a transverse wave propagating in the $z$-direction. The subscript $A T$ refers

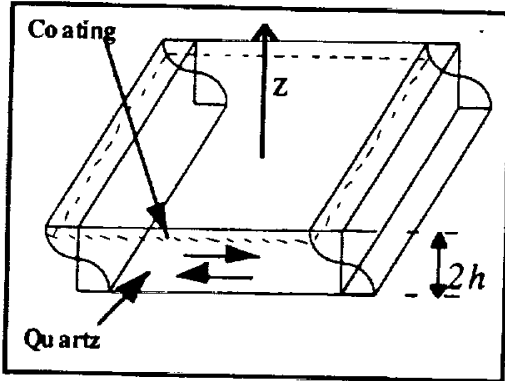

Figure 1: Planar resonator and thickness-shear mode

to the specific orientation of the cut of the plate resonator with respect to the crystal axes. ${ }^{11}$ The AT-cut is a special case of orientation that ensures oscillations in the thickness-shear mode, while exhibiting very small frequency changes due to temperature variations $( \pm .3 \mathrm{~Hz} / \mathrm{K}){ }^{13}$

Introducing $\rho_{Q}$, the quartz density, and substituting into Eq. (I) we have

$$
\Delta \sigma=\frac{\rho_{Q} C_{\Lambda T} / 2}{f_{0}^{2}}\left(f_{0}-f\right)
$$

where $f$ is the frequency after the shift due to the loading. For a uniform layer on an infinite plate we therefore have

$$
\Delta f=-C_{f} \Delta \sigma
$$

where

$$
C_{f}=\frac{f_{0}^{2}}{\rho_{Q} C_{A T} / 2}
$$

is the sensitivity constant for the resonator. With $f_{0}=6$ $\mathrm{MHz}, \rho_{Q}=2.649 \mathrm{~g} / \mathrm{cm}^{3}$ and $C_{A T}=3322 \mathrm{~m} / \mathrm{s}$, the values corresponding to the crystal in this study, ${ }^{13,14}$ the sensitivity constant $C_{f}$ is $8.18 \times 10^{7} \mathrm{~Hz} . \mathrm{cm}^{2} / \mathrm{g}$ so that the removal of a uniform layer of $1 \AA$ of molybdenum would correspond to a increase in the resonant frequency of $8.35 \mathrm{~Hz}$, about a hundred times the theoretical resolution of the instrument.

As mentionned earlier in this part however, it is not always practical to have to work with a uniform layer. In addition, for a circular, plano-convex, finite (with boundary conditions) resonator loaded with a point mass $\Delta m$ (Fig. 2), Eq. (4) becomes ${ }^{12.13}$

$$
\Delta f=-c_{f}(r, \theta) \Delta m
$$



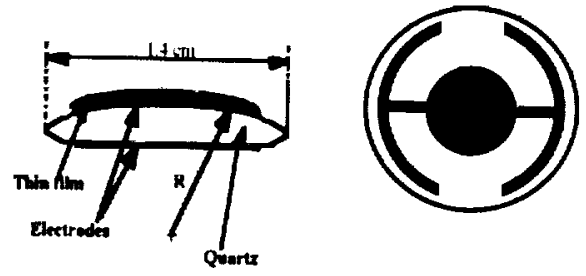

Figure 2: Plano-convex resonator. Edge view (left) and exciting electrode or bottom view (right)

where $c_{f}(r, \theta)$ is the differential mass sensitivity and $r$, $\theta$ are the local radial and azimutal coordinates, with the axis $\theta=0$ oriented along the crystallographic $x$-direction. The mass sensitivity constant $C_{f}$ is now given by the integral over the crystal surface area $S$, of the differential mass sensitivity $c_{f}$ as expressed below

$$
C_{f}=\iint_{s} c_{f}(r, \theta) d s
$$

Eq. (6) is an important result, as it shoes that knowledge of the differential mass-sensitivity allows the QCM to be applied to the measurement of masses known to be distributed unevenly.

Cumpson and Seah ${ }^{13}$ performed a thorough theoretical analysis of the response of AT-cut quartz crystal microbalances and presented the following analytical solution for the radial and polar dependence of mass-sensitivity

$$
c_{f}(r, \theta)=c_{0} e^{-r^{2}\left(\alpha_{1} \cos ^{2} \theta+\beta_{1} \sin ^{2} \theta\right)}
$$

where

$$
c_{0}=\frac{f_{0}^{2}\left(\alpha_{1} \beta_{1}\right)^{1 / 2}}{\pi \rho_{Q} C_{A T} / 2}
$$

and

$$
\begin{aligned}
& \alpha_{1}^{2}=\frac{\pi^{2} \bar{C}_{66}}{8 R h_{0}^{3} M_{1}}\left(1-\frac{8 k_{26}^{2}}{\pi^{2}}-\frac{2 \rho_{E} h_{E}}{\rho_{Q} h_{0}}\right) \\
& \beta_{1}^{2}=\frac{\pi^{2} \bar{C}_{66}}{8 R h_{0}^{3} C_{55}}\left(1-\frac{8 k_{26}^{2}}{\pi^{2}}-\frac{2 \rho_{\varepsilon} h_{E}}{\rho_{Q} h_{0}}\right)
\end{aligned}
$$

In the above expressions, $M_{1}, k_{26}^{2}, C_{55}$ and $\bar{C}_{66}$ are quantities derived from the elastic modulus tensor and the piezoelectric coupling constants of quartz, and have values $^{15}$

$$
\begin{aligned}
& \bar{C}_{06}=2.924 \times 10^{10} \mathrm{~N} \mathrm{~m}^{-2} \\
& C_{55}=6.881 \times 10^{10} \mathrm{~N} \mathrm{~m}^{-2} \\
& M_{1}=1.10 \times 10^{11} \mathrm{~N} \mathrm{~m}^{.2}
\end{aligned}
$$

$$
k_{26}^{2}=7.744 \times 10^{-3}
$$

$h_{E}$ and $\rho_{E}$ are the electrode (coating) material thickness and density, and $R$ is the curvature of the plano-convex crystal surface, $0.212 \mathrm{~m}$ ( 2.5 diopters) here.

Fig. 3 shows a plot of $c(r, \theta)$. Since the crystal is anisotropic (i.e. $\alpha_{l}$ and $\beta_{l}$ are different), the plot is a gaussian stretched in the $x$-direction. As shown by Cumpson and Seah, ${ }^{13}$ the differential masssensitivity at any point is proportionnal to the square of the vibration amplitude at that point, so that Fig. 3 also shows that, due to the convexity of the resonator surface and the small size of the exciting electrode (Fig. 2), the oscillation energy is trapped in the center and very little dissipation occurs at the edge. This explains why the quality factor of such resonators is so large and therefore the resonant frequency so stable, and justifies that the crystal can be clamped at the edge, where a small wire can be put in contact with the coated electrode to collect an impinging current.

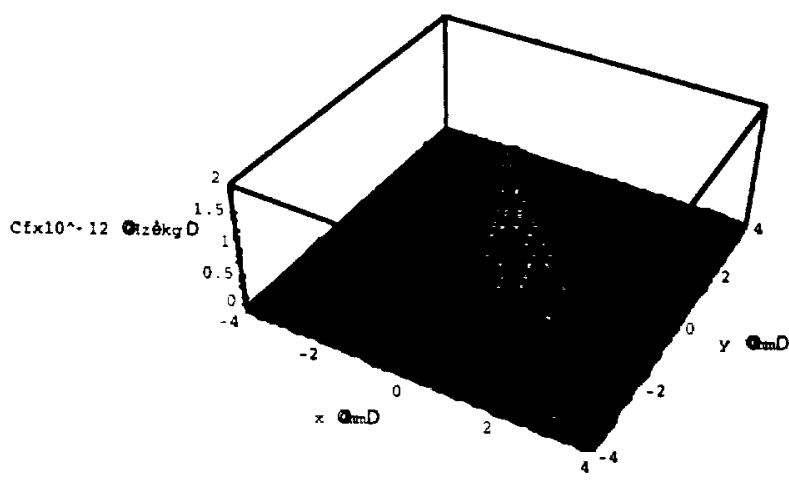

Figure 3: Differential mass-sensitivity $c f(r, q)$ as a function of position on a plano-convex piezoelectric quartz crystal resonator

As a final note, it should be added that the electronic equivalent of the crystal resonator is roughly equivalent to a sharply-tuned LCR circuit. ${ }^{10}$ The resonance is found by the instrument by comparing the phase of the current driving the electromechanical oscillator to that of the current passing through the crystal, which should be zero at resonance, i.e. the crystal behaves as a pure resistance. The fact that the operation of the instrument relies on the phase of the current passing between the electrodes of the crystal explains why a DC current on the coated electrode from an ion beam does not interfere with the operation of the instrument.

\section{Experimental}




\section{Vacum System}

The experiments were conducted in a small vacuum chamber (Fig. 4) consisting primarily of a 117.5-mm (4-5/8") six-way cross fitted with conical reducers. The vacuum chamber is bolted on a Pfeiffer TPU 270 turbomolecular pump and evacuated to a total base pressure in the low $10^{-6}-\mathrm{Pa}\left(10^{8}\right.$-Torr) range after bakeout. A low base pressure was an important requirement driven by the necessity to keep the sample surface as free as possible from adsorbed impurities or oxydation. This critical issue is discussed at greater lengths in part 4

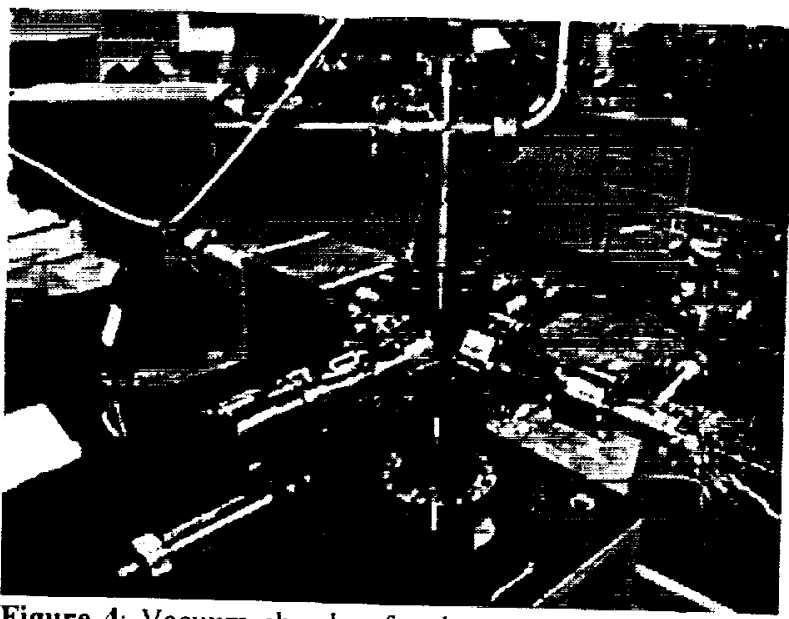

Figure 4: Vacuum chamber for the experiments.

\section{Iongun}

The ion gun used in the experiments described here is the ILG-2 ion gun built by Kimball Physics Inc, and has a beam energy adjustable from $10 \mathrm{eV}$ to $1 \mathrm{keV}$, with total beam current on xenon usually of the order of $100 \mathrm{nA}$. The beam can be focused to a spot size (containing 95\% of the current) less than $5 \mathrm{~mm}$ in diameter on the sample, which was positionned $1 \mathrm{~cm}$ away from the ion gun. The energy spread varied from $20 \mathrm{eV}$ down to $4 \mathrm{eV}$ for a beam energy of $1 \mathrm{keV}$ and $100 \mathrm{eV}$, respectively. The energy stability (from the power supply specifiations) was $0.01 \%$.

The ion gun was differentially-pumped using a VEECO MS-20T leak detector equiped with a Pfeiffer TPU 050 tubomolecular pump providing a net pumping speed of the order of a liter per second in the source region.

\section{Beam diagnostics}

The ion velocity distributions shown in $\mathbf{F i g}$. 2 were obtained using a velocity filter (ExB probe) designed and built specificilly for this experiment. The ExB probe was used to characterize the energy spread of the ion source and to determine the doubly-to-singly charged ions ratio. Fig. 3 shows ExB traces for $400-$ $\mathrm{eV}$ xenon ions as a function of electron energy, or discharge voltage. In the xenon-ion kinetic energy range considered here, a discharge voltage of $50 \mathrm{~V}$ was chosen to optimize the beam current and the doubly-to-singly charged ion current ratio, equal to $5 \%$.

Beam current density profiles were obtained for each run at the corresponding ion gun and pressure settings by means of a small Faraday cup with a $2-\mathrm{mm}$ circular aperture. In addition, the current hitting the 8$\mathrm{mm}$ diameter sample at the center of the beam, as well as the current hitting a $34-\mathrm{mm}$ circular graphite mask around it were measured and recorded at all times.

\section{Sputtering diagnostics}

The Quartz Crystal Microbalance (QCM) technique, discussed in detail in part 1, was used to measure the amount of molybdenum etched from the sample by the xenon ion bombardment. The QCM sensor, holding the quartz crystal resonator coated with a sputter-deposited film of molybdenum, could be moved in the direction perpendicular to the beam by means of a linear motion feedthrough. The motion feedthrough itself was mounted on a flexible coupling to allow for gross positioning and alignment corrections (see Fig. 1).

As shown in Fig. 4 and in order to be able to monitor the portion of beam current infringing on the molybdenum sample, the QCM sensor and crystal holder were modified to break the electrical continuity between the molybdenum-coated electrode of the quartz crystal and the crystal holder (ground). The coated electrode was then grounded through a picoammeter. In addition, a 34-mm graphite mask was mounted on the crystal holder to protect the whole assembly from ionimpingement erosion. The beam current collected by this mask was also monitored. An electrical schematic of the QCM sensor assembly is shown in Fig. 5. The arrangement was very similar to the one described in References? and 8.

\section{Qthers}

An SRS RG200 Residual Gas Analyzer (RGA) mounted on the chamber and a T-type thermocouple junction in contact with the crystal holder completed the diagnostics. In addition, a circulating bath was used to regulate the $\mathrm{QCM}$ sensor temperature within $\pm 0.2^{\circ} \mathrm{C}$ at room temperature.

\section{Data Acquisition}

All parameters monitored during the sputtering experiments were averaged over 200 to 500 measurements depending on the sampling period. The 
recorded parameters on fifteen channels were: elapsed time; ten ion gun parameters including ion energy; mask current; sample current; crystal holder temperature; and sample thickness returned by the QCM monitor. The signals were processed by National Instruments 5B-series analog signal conditioning modules with $\pm 5-\mathrm{V}$ outputs and acquired by a computer running LabView via a National Instruments NB-MIO16XL-18 DAQ board. The RGA trace was recorded on a separate computer.

\section{Procedure}

The following sequence was followed when running an experiment: 1) Turn on the cooling system and wait for the temperature to stabilize; 2) Select the ion gun operating point; 3) Establish the proper xenon pressure of $\sim 1.5 \times 10^{-6} \mathrm{~Pa}\left(2 \times 10^{-4}\right.$ Torr $)$ in the source region of the ion gun; 3 ) Turn on the ion gun to the selected operating point; 4) Acquire a current density profile versus radial position across the ion beam with the Faraday probe, noting the exact position of the center of the beam; 5) Place the center of the coated crystal electrode at that position and start recording the parameters; 6) After a measurable amount of material has been eroded, turn off the ion gun and keep recording for a time comparable to the duration of exposure.

For experiments at ion energies below $500 \mathrm{eV}$ and at the beginning of step 5, the sample was sputtercleaned at $1 \mathrm{keV}$ with a broader beam until at least $10 \AA$ were removed before setting the energy and focusing back to the previous levels from step 3. In this case, another beam current density profile was acquired at the end of the experiment to check that it had been left unchanged. The ion gun operating points were found to be quite repetable however. For ion energies at or above $500 \mathrm{eV}$, the data corresponding to the first $10 \AA$ (approximately the first ten monolayers) were ignored in the sputtering yield calculations, to account for possible sputtering of surface impurities.

Step 6 was performed to check that the observed erosion was solely due to the ion beam impingement and that sputtering was not competing with any comparable regenerative process at the surface, like adsorption.

\section{Molybdenum samples}

\section{Preparation}

The preparation of the molybdenum samples was determined by two sets of constraints.

The first constraints were, understandably, dictated by the need to perform the measurements on material samples representative of the material on an actual ion engine. This meant that the molybdenum samples should have material properties similar to the molybdenum used for the ion optics, at least for those properties likely to affect the sputtering yield: density and priviledged directions, e.g. in the case of a crystaline structure. Possible residual stresses in the molybdenum film, unless they resulted in a problem of adhesion, were not believed to be likely to affect sputtering, since the surface would remain stress-free and sputtering, even more so at low energy, is essentially a surface process.

Scanning Electron Microscope (SEM) micrographs were obtained on molybdenum grid bits (Fig. 6), either from the surface at various locations in the erosion pattern, or from an edge. For the latter case, the grid bit was either broken in liquid nitrogen, to ensure a brittle fracture, or was laser-cut and/or mechanically and chemically polished. The micrographs did not reveal any distinguishable microstructure. Rather, a laminar pattern with length scale $1 \mu \mathrm{m}$ was apparent, likely to be the result of the lamination in the manufacturing process of the molybdenum sheets used for the grids.

The second set of contraints resulted from the choice of the QCM technique to perform the measurements. Due to its extreme sensitivity to areal mass density $\left(8.18 \times 10^{7} \mathrm{~Hz} / \mathrm{g} / \mathrm{cm}^{2}\right)$ and finite frequency range $(6-5 \mathrm{MHz})$, only a limited amount of material can be used to load the surface of the quartz crystal (equivalent to a $12-\mu \mathrm{m}$ molybdenum film).

Two schemes were considered to prepare the samples. The first one was to bond a $4-\mu \mathrm{m}$ molybdenum foil on the crystal electrode, while the second method was sputter deposition.

The thin film bonding method disallowed the use of an epoxy-type glue, as the bond line would have been excessively thick (over $100 \mu \mathrm{m}$ ). Two samples were prepared using a cyano-acrylate glue with a bond line thickness of about $1 \mu \mathrm{m}$, and the shear wavespeed, necessary to correct for the acoustic impedance of the glue in the QCM monitor, was measured to be about $816 \mathrm{~m} / \mathrm{s}$ for a density of about $1 \mathrm{~g} / \mathrm{cm}^{3}$. The crystal oscillations, however, had a tendency to be erratic, due either to that rather dissipative additionnal layer or to bad adhesion under vacuum. The first method was therefore quickly abandoned.

Sputter-deposition of molybdenum on the crystal oscillator was then chosen as the method of choice.

The morphology of a metal coating deposited by sputtering is best described as a function of working pressure and temperature by a Thornton diagram, ${ }^{5,6}$ as reproduced in Fig. 7. Bulklike properties can best be obtained when the structure is that of the zone $T$, or 
transition zone, consisting of a dense array of poorly defined fibrous grains ${ }^{6}$ with a slightly compressive stress. As seen on the Thornton diagram, such a structure is obtained by sputtering preferably at a low pressure (of the order of 0.1-1 Pa, or 1-10 mTorr) and intermediate temperatures a few tenth of a percent the melting temperature of the metal. Since planar diode sputtering typically requires pressures 10 to 100 times higher to achieve a satisfactory ionization efficiency, the low pressure requirement mandated the use of magnetron sputtering, and while it was impractical to heat the samples in the deposition chamber, the samples where biased negatively to $-80 \mathrm{~V}$. This negative bias, while insufficient to induce significant implantation of working gas ions in the sample, had the effect of densifying the microstructure by filling the voids due to enhanced mobilty and forward sputtering.

Finally, the density of a thin film can be greatly affected by its thickness, as shown for aluminum $^{8}$ in Fig. 8. In this example, the density reaches $4 \%$ of the bulk density at $2000 \AA(0.2 \mu \mathrm{m})$. Problems of thermal expansion mismatch can arise however for excessively large thicknesses, and the molybdenum samples deposited on the gold electrode of the crystal resonator for this study had a thickness of 3 $\mu \mathrm{m}$. Additional samples were prepared with different thicknesses, bias voltages and materials including tantalum and tungsten, and will be used in further studies. Table 1 summarizes the deposition parameters for the molybdenum sample used here. Fig. 9 shows the film structure obtained under different anode bias voltages, and Fig. 10 details the surface morphology.

Table 1: magnetron sputtering deposition parameters

\begin{tabular}{|ll|}
\hline Working gas & Argon \\
Working pressure & $1.3 \mathrm{~Pa}(10 \mathrm{mTorr})$ \\
Temperature & $-100^{\circ} \mathrm{C}(13 \%$ melting temp.) \\
Bias voltage & $-80 \mathrm{~V}$ \\
Deposition rate & $670 \mathrm{~A} / \mathrm{min}$ \\
\hline
\end{tabular}

Density

The density of the samples was estimated in four different ways as described below, and as summarized in Table 2.

A silicon substrate used as a witness was coated with molybdenum during each sputter-deposition. A mask was placed to cover a small fraction of their surface area so that the thickness of the deposited molybdenum film could be measured by means of a stylus profilometer, with the errors shown in Table 2 . In addition, a few molybdenum samples were deposited with thicknesses $2.5,5$ and $10 \mathrm{kA}$, a thickness range where Rutherford Backscattering Spectrometry (RBS) could be used to determine the sample density from the known thicknesses.

A variation of this method was to use the RBS data in conjunction with the RBS-simulation software "Rump" to obtain the density by curve-fitting, again from the known thickness.

The third method was to place the molybdenum-coated crystals with known thicknesses in the quartz crystal sensor and infer the density from the frequency shift, measured with an accuracy better than one part per million. This method was applied only to the $30-\mathrm{k} \AA$ samples.

Finally, the mass difference of the crystals before and after deposition was also used to provide an estimate of the target densities, as shown in Table 2 . It should be noted however that all four methods relied on the knowledge of the thickness of the deposition.

Table 2: density estimates for the sputter-deposited molybdenum films

\begin{tabular}{|c|c|c|c|}
\hline Method & $\begin{array}{l}\text { Number of } \\
\text { samples }\end{array}$ & $\begin{array}{l}\text { Properties used } \\
\text { and } \\
\text { associated error }\end{array}$ & $\begin{array}{l}\text { Density } \\
{\left[\mathrm{g} / \mathrm{cm}^{3}\right]}\end{array}$ \\
\hline RBS & $\begin{array}{l}1 \\
1 \\
1 \\
\end{array}$ & $\begin{array}{l}2.5 \mathrm{k} \AA \pm 4 \% \\
5.2 \mathrm{kA} \pm 5 \% \\
10.3 \mathrm{k} \AA \pm 2.5 \% \\
\end{array}$ & $\begin{array}{l}10.9 \pm 4 \% \\
10.6 \pm 5 \% \\
10.7 \pm 2.5 \% \\
\end{array}$ \\
\hline $\begin{array}{l}\text { RBS + } \\
\text { Rump }\end{array}$ & & $\begin{array}{l}2.5 \mathrm{k} \AA \pm 4 \% \\
5.2 \mathrm{kA} \pm 5 \% \\
\end{array}$ & $\begin{array}{l}10.8 \pm 4 \% \\
10.2 \pm 5 \% \\
\end{array}$ \\
\hline$Q C M$ & $\begin{array}{l}5 \text { ( } 80-\mathrm{V} \text { bias }) \\
4 \text { (150-V bias) }\end{array}$ & $\begin{array}{l}30 \mathrm{kA} \pm 3 \% \\
30 \mathrm{kA} \pm 3 \% \\
\end{array}$ & $\begin{array}{l}13.6 \pm 3 \% \\
14.1 \pm 3 \% \\
\end{array}$ \\
\hline $\begin{array}{l}\text { Weight } \\
\text { gain }\end{array}$ & $\begin{array}{l}6 \text { ( } 80-\mathrm{V} \text { bias }) \\
4 \text { (150-V bias) }\end{array}$ & $\begin{array}{l}30 \mathrm{kA} \pm 3 \% \\
4.95 \mathrm{mg} \pm 10 \% \\
30 \mathrm{kA} \pm 3 \% \\
6.22 \mathrm{mg} \pm 8 \%\end{array}$ & $\begin{array}{l}10.7 \pm 13 \% \\
13.5 \pm 11 \%\end{array}$ \\
\hline
\end{tabular}

All four methods seemed to point to a value slightly higher than the bulk density of $10.22 \mathrm{~g} / \mathrm{cm}^{3}$ (which is unrealistic), while being within at most a few percent. Although this value is not critical for the QCM technique since only relative measurements of thickness changes are made here, density is a important indicator of thin film morpholgy and care had to be taken to prepare bulk-like samples for the sputtering experiments. It should also be pointed out that the RBS spectra revealed a level of impurity limited to 0.1 to 0.2 percent (identified as tungsten), which was qualitatively confirmed with SEM elemental analysis.

\section{Target contamination}

Yonts and Harrison ${ }^{5}$ have discussed the competing processes involved in the building and destruction of the surface layer on a target bombarded by an ion beam in an ultra-high vacuum system. Their 
simplified analysis describes the sticking process by the equation

$$
\frac{\partial n}{\partial t}=A n_{G}-n_{E}
$$

where $n$ is the surface layer density in atoms per unit area, $n_{G}$ is the number of reactive gas atoms striking a unit surface per second and $A$ is the corresonding sticking probability, such that $A n_{G}$ is the number of atoms sticking to a unit area per second. $n_{E}$ is the number of atoms emitted per second from a unit area. The authors showed that the evolution of the surface layer with time is then, after integration, given by

$$
n=n_{0} e^{-D t}+(A / D) n_{G}\left(1-e^{-D t}\right)
$$

where $D$ can be written as

$$
D=\frac{J Y}{e}
$$

and $n_{0}$, the initial layer, is destroyed. $J$ is the beam current density, $e$ the proton charge and $Y$ the sputtering yield of the emitted atoms. The equilibrium layer, or limit layer, can therefore be expressed as

$$
n_{\infty}=(A / D) n_{g}=A(e / Y)\left(n_{G} / J\right)
$$

$n_{g}$ is proportional to $p /(M T)^{1 / 2}$ where $p$ is the pressure, $M$ the molecular mass and $T$ the temperature, as given by the kinetic theory of gases. This result can be generalized ${ }^{6}$ for multiple species by stating that

$$
n_{\infty}=\sum_{i} \frac{A_{i, s} n_{G_{i}}}{Y_{i, s} n_{l}}
$$

where $A_{i, s}=$ sticking probability of back- ground gas species $i$ on a clean target $s$

$$
Y_{i, s}=\text { sputtering yield of species } i \text { adsorbed }
$$
on target $s$

$n_{G i}=$ particle flux density of residual

gas species $i$

$n_{l}=$ beam particle flux densit

The balance between the regenerative effect of the system residual gas and the destructive effect of the beam appears directly in the ratio of $n_{g}$ to $n_{r}$. The difficulty resides in the determination of $A_{i, s}$, the sticking probabilities, and $Y_{i, s}$, the sputtering yields, of the particles making up the adsorbed or chemisorbed surface layer. In particular, the sputtering yield of a species for a few monolayers deposited on a target of a different material is in general different than the bulk sputtering yield for this species.

\section{Results and discussion}

\section{Data interpretation}

As explained in part 1, the QCM operates by sensing a shift in the resonant frequency of a single crystal piezoelectric quartz resonator. The frequency information is then converted to a measured thickness change $\Delta t_{\text {meas }}$ sent as an output by the QCM monitor to the data acquisition. That measured thickness change, however, does not account for the non-unformity of the sputerring over the sample surface area, due to the nonuniform ion beam current density profile.

The sputtering yield $Y$ at any radial position $r$ on the sample is given by

$$
Y=\frac{\text { number of atoms removed }}{\text { number of incident ions }}
$$

which is written

$$
Y=\frac{\Delta t(r) x d s \times n}{\Delta \tau x \frac{J(r)}{e} x d s}
$$

where $\Delta t(r)$ is the local thickness change, $d s$ is an elementary surface area, $n$ is the atomic number density of the sample material, $\Delta \tau$ is the time of exposure, $J(r)$ is the local beam current density, and $e$ is the electronic charge.

The measured thickness change $\Delta t_{\text {meas }}$ is a surface-average of the local thickness change over the sensor area. The crystal differential mass-sensitivity, $c(r, \theta)$, is the weighing function for this average, since from Eqs. (4) and (6) we have

$$
C_{f} \Delta t_{\text {meas }} \rho_{c}=\iint_{s} c_{f}(r, \theta) d m
$$

where $\rho_{c}$ is the coating material density, and this in turn leads to

$$
\Delta \Delta_{\text {meas }}=\frac{1}{C_{\digamma}} \iint_{s} \Delta t(r) c_{f}(r, \theta) d s
$$

where $\mathrm{Cf}$ is given by Eq. (6). From Eq. (*2) then

$$
\Delta \delta_{\text {mers }}=\frac{1}{C_{f}} \iint_{s} c_{f}(r, \theta)\left[Y_{x} \Delta \tau \frac{J(r)}{n e}\right] d s
$$

Finally, the sputtering yield is given by 


$$
Y=\frac{\Delta t_{\text {mens }} x n e C_{f}}{\Delta \tau x \int_{0}^{a} \int_{0}^{2 \pi} c_{f}(r, \theta) J(r) r d r d \theta}
$$

In this expression, $a$ is the radius of the sensor active area, and the integral over the surface area in the denumerator of the right-hand side will be called the Current-Sensitivity Integral (CSI). The CSI represents the effective average beam current density for the sensor, and becomes larger as the beam is focused to a smaller spot at the center of the electrode due to the gaussian nature of $c(r, \theta)$. The benefit of focusing was in fact twofold, as the total beam current from the ion gun tends to increase with focusing voltage. It should however be noted that an excessively focused beam results in a greater alignment sensitivity. A moderate focusing was therefore chosen in these experiments, keeping the spot size for $95 \%$ of the beam current to about $4 \mathrm{~mm}$.

\section{Results}

As mentioned in the description of the experimental procedure, the beam profile $J(r)$ was obtained for each experiment and fit to a gaussian curve, with good agreement, to calculate the CSI. Figs. 14 and 15 show both the data and the gaussian fit for the beam current density profile across a $1-\mathrm{keV}$ and $200-\mathrm{eV}$ beam, respectively.

Similarly, a linear fit was performed on the thickness measurement $\Delta t_{\text {meas, }}$ with the first $10 \AA$ disregarded for sputter-cleaning, as explained in the procedure. Figs. 16 and 17 show both the data and the fit for the $1-\mathrm{keV}$ and $200-\mathrm{eV}$ ion beams, respectively.

Using the CSI and the slope of the linear fit of $\Delta t_{\text {meas }}$ versus time (or $\Delta t_{\text {meas }}$ and $\Delta \tau$ ), the sputtering yield $Y$ for the corresponding energy was then obtained using Eq. (*6).

The recorded ion parameters, graphite mask current, sample current and sensor temperature were checked for stability. The measured sample current was the superposition of the beam current hitting the sample (between ? and $25 \mathrm{nA}$ ), the secondary electron current and the current flowing between the crystal electrodes as a result of the QCM operation (about $12.5 \mu \mathrm{A}$ ). Since the QCM current is three orders of magnitude larger than the beam current with small-scale variations of $\pm 10 \mathrm{nA}$, this data was only used qualitatively to check that the current was held to the required level of stability during the experiements. The jump in current when the beam was turned on or off was noted but did not agree with the integrated beam current density profile however. Future work will focus on modeling the response of the electronic analog of the quartz crystal to remove the QCM current. The correction for the secondary-electron emission current for molybdenum at xenon ion energies between $1 \mathrm{keV}$ and $100 \mathrm{eV}$ was $1 \%$ at $\mathrm{l} \mathrm{keV}$ and below at lower energies." The secondary electron emission could therefore be ignored. Similarly, the current collected from the graphite mask was useful in checking the stability of the beam but did not agree with the corresponding integral of $J(r)$. This, however, was less surprising as the gaussian fit was not satisfactory at the corresponding radial positions (beyond $\pm 4 \mathrm{~mm}$ ). This however was not important for the yield measurements.

Since the quartz resonator is known to be sensitive to temperature, the sample current, largely dominated by the QCM current, was found to mirror very closely any change in temperature, e.g. when the beam was turned on or off. Large time-scale temperature changes were corrected by an amount of $\pm 3 \mathrm{~Hz} / \mathrm{K}$, while the short time-scale thermal noise was propagated into the error analysis in Eq. (*6). Finally, a conservative misalignment uncertainty of $\pm 1 \mathrm{~mm}$ was assumed in the error analysis to calculate the error on the CSI. Finally, the experiment at $100 \mathrm{eV}$ carries a larger error on the sample current, as the beam was less stable. This problem will be addressed in future work as the ion kinetic energy will be decreased.

Table 3 is a summary the results where the calculated errors were indicated using the assumptions above. The sample current is the integral of $J(r)$ between $\pm 4 \mathrm{~mm}$ (aperture diameter) as measured by the Faraday cup, while the mask current is the current collected by

Table 3: Summary of the results for xenon ions on molybdenum

\begin{tabular}{|lllll|}
\hline $\begin{array}{c}\text { Energy } \\
(\mathrm{eV})\end{array}$ & $\begin{array}{c}\text { Sample } \\
\text { Current } \\
(\mathrm{nA})\end{array}$ & $\begin{array}{c}\text { Mask } \\
\text { current } \\
(\mathrm{nA})\end{array}$ & $\begin{array}{c}\text { CSI } \\
\left(\mathrm{nA} / \mathrm{mm}^{2}\right)\end{array}$ & $\begin{array}{c}\text { Sputter } \\
\text { yield } \\
\text { (aVion) }\end{array}$ \\
\hline 1000 & & 8.2 & 19.06 & 1.53 \\
900 & & 8.1 & 12.10 & 1.97 \\
800 & & 4.9 & 30.80 & 1.48 \\
700 & & 16.7 & 12.14 & 1.13 \\
600 & & 14.1 & 19.20 & 0.90 \\
500 & 59.54 & 32.1 & 10.24 & 0.72 \\
400 & 59.99 & 31.9 & 10.60 & 0.73 \\
300 & 61.45 & 21.3 & 13.26 & 0.50 \\
250 & 44.98 & 41.1 & 7.62 & 0.23 \\
200 & 41.85 & 40.4 & 7.09 & 0.14 \\
100 & 25.05 & 42.5 & 3.41 & 0.08 \\
\hline
\end{tabular}

the graphite shield. The effect of focusing on the effective average current density is emphasized with the experiments at $800 \mathrm{eV}$ (high focusing) and below 300 $\mathrm{eV}$ (low focusing). Table 4 compares the results with previously published data in the same energy range. Finally, Fig. 19 is a plot of the data in Table 4 , 
along with the most commonly used semi-empirical formulae to the energy dependence of the sputtering yield.

Table 4: comparison with data from published litterature, xenon ions on molybdenum

\begin{tabular}{|cccccc|}
\hline $\begin{array}{c}\text { Energy } \\
(\mathrm{eV})\end{array}$ & $\begin{array}{c}\text { Ref. 1 } \\
\text { Weisj }\end{array}$ & $\begin{array}{c}\text { Ref. 2 } \\
\text { R\&W }\end{array}$ & $\begin{array}{c}\text { Ref. 2 } \\
\text { Bland }\end{array}$ & $\begin{array}{c}\text { Ref. 3 } \\
\text { Batt }\end{array}$ & $\begin{array}{c}\text { Present } \\
\text { study }\end{array}$ \\
\hline 1000 & 1.6 & - & - & - & 1.53 \\
900 & 1.42 & - & - & - & 1.97 \\
800 & 1.24 & - & - & - & 1.48 \\
750 & - & - & 0.61 & - & - \\
700 & 1.06 & - & - & - & 1.13 \\
600 & 0.88 & 1.06 & - & 0.78 & 0.90 \\
500 & 0.69 & - & 0.55 & 0.70 & 0.72 \\
400 & 0.6 & 0.7 & - & 0.65 & 0.73 \\
300 & 0.4 & 0.51 & - & 0.60 & 0.50 \\
250 & - & - & 0.20 & - & 0.23 \\
200 & 0.2 & 0.28 & - & 0.30 & 0.14 \\
150 & - & - & 0.13 & 0.09 & - \\
100 & 0.023 & 0.06 & - & - & 0.08 \\
\hline
\end{tabular}

\section{Conclusion}

The QCM technique was found to enable distinctive and consistent detection of the removal of less than an atomic monolayer of molybdenum at the surface of a $3-\mu \mathrm{m}$ molybdenum sample deposited on a piezoelectric quartz crystal electrode by magnetron sputtering with an anode bias of $-80 \mathrm{~V}$.

The preliminary results presented in this paper are found to be in satisfactory agreement with previously published data, so that the authors feel confident that the method can be extended to other metallic materials and ion kinetic energies closer to the energy threshold for sputtering by xenon-ion bombardment. Remaining issues to be addressed are primarily 1) The use of the coated electrode as a probe of the beam current once the QCM current will have been accurately modeled; 2 ) Improved vacuum chamber base pressure.

\section{Acknowledgments}

The work described in this paper was performed by the Jet Propulsion Laboratory (JPL), California Institute of Technology, under contract with the National Aeronautics and Space Administration. The authors wish to thank Dr. E. Kolawa (JPL) and Dr. S. Gasser (Caltech) for use of the magnetron sputtering laboratory and help with the depositions. Grateful acknowledgments are also due to J. Blandino (JPL) for critical discussions and insight for improvements.

\section{References}

1. Weijsenfeld, C. H., Hoogendoorn, A., and Koedam, M., "Sputtering of Polycrystalline Metals by Inert Gas Ions of Low Energy (100-1000 eV)," Physica 27, 763 (1961).

2. Rosenberg, D., and Wehner, G. K., "Sputtering Yields for Low Energy $\mathrm{He}^{+}-, \mathrm{Kr}^{+}$-, and $\mathrm{Xe}^{+}-\mathrm{Ion}$ Bombardment," J. of Appl. Phys. 33 (5), 1842 (1962).

3. Stuart, R. V., and Wehner, G. K., "Sputtering Yields at Very Low Bombarding Ion Energies," J. of Appl. Phys. 33 (7), 2345 (1962).

4. Bhattacharjee, S., Zhang, J., Shutthanandan, V., Ray, P. K., Shivaparan, N. R., Smith, R. J., "Application of Secondary Neutral Mass Spectrometry in Low-Energy Sputtering Yield Measurements," Nucl. Instr. and Meth. in Phys. Res. B129, 123 (1997).

5. McKeown, D., "New Method for Measuring Sputtering in the Region near Threshold," The Rev. of Scientific Instruments 32 (2), 133 (1960).

6. Hayward, W. H., and Wolter, A. R., "Sputtering Yield Measurements with Low-Energy Metal Ion Beams," J. of Appl. Phys. 40 (7), 2911 (1968).

7. Andersen, H. H., and Bay, H., "The ZI Dependence of Heavy-Ion Sputtering Yield in Copper," Rad. Eff. 13, 67 (1972).

8. Fine, J., "Absolute Sputtering Yield Measurement Methods: A Review," Physics of lonized Gases. Invited Lectures, Matic, M., Ed. Boris Kidric Institute, Beograd, 379 (1980).

9. Duchemin, O. B., Brophy, J. R., Garner, C. E., Ray, P. K., Shutthanandan, V., and Mantenieks, M. A., "A Review of Low-Energy Sputtering Theory and Experiments," IEPC 97-068, presented at the 25th International Electric Propulsion Conference, Aug. 24-28 1997, Cleveland, OH.

10. Gogol, C. A., "Advances in the Quartz Crystal Deposition Controller, A History of the Technology and Recent Technical Improvements," Leybold Inficon Inc., East Syracuse, NY, personnal communication (1990).

11. Lu, C., and Czanderna, A. W., Ed. Applications of Piezoelectric Quartz Crystal Microbalances, Methods and Phenomena, their Applications in Science and Technology, Vol. 7, Elsevier (1984).

12. Sauerbrey, G., "Use of Oscillator Quartz Crystals for Weighing Thin Layers and Microweighing," Zeitschrift für Physik 155, 206 (1959).

13. Cumpson, P. J., and Seah, M. P., "The Quartz Crystal Microbalance; Radial/Polar Dependence of 
Mass Sensitivity Both On and Off the Electrodes," Meas. Sci. Technol. 1, 544 (1990).

14. XTM/2 Deposition Monitor Manual, Leybold Inficon Inc., PN 074-186, East Syracuse, NY (1996).

15. Tiersten, H. F., and Smythe, R. C., "Analysis of Contoured Crystal Resonators Operating in Overtones of Coupled Thickness-Shear and Thickness-Twist," J. Acoust. Soc. Am. 65 (6), 1455 (June 1979).

16. Vossen, J. L., and Kern, W., Ed. Thin Film Processes, Academic Press, Inc. (1978), p. 106.

17. Thornton, J. A., J. of Vac. Sci. Technology 11 , 666 (1974).

18. Belkind, A., Thin Film Phenomena, an Introductory Course, International Conference on Metallurgical Coatings and Thin Films (1998), San Diego, CA.

19. Chopra, K. L., Thin Film Phenomena, McGrawHill (1969).

20. Yonts, O. C., and Harrison, D. E. Jr., "Surface Cleaning by Cathode Sputtering," J. of Appl. Phys. 31 (9), 1583 (1960).

21. Behrisch, R., Sigmund, P., Robinson, M. T., Andersen, H. H., Bay, H. L., and Roosendaal, H. E., Sputtering by Particle Bombardment I: Physical Sputtering of Single-Element Solids, Topics in Applied Physics 47, Springer-Verlag (1981).

22. Brown, S. C., Basic Data of Plasma Physics, 2nd Ed., MIT press p. 232 (1966). 